\title{
Selective Surface and Near-Surface Modification of a Noncatenated, Catalytically Active Metal-Organic Framework Material Based on Mn(salen) Struts
}

\author{
Abraham M. Shultz, Omar K. Farha, Debashis Adhikari, Amy A. Sarjeant, Joseph T. Hupp,* and \\ SonBinh T. Nguyen* \\ Department of Chemistry and Institute for Catalysis in Energy Processes, Northwestern University, 2145 Sheridan Road, Evanston, \\ Illinois 60208, United States
}

Supporting Information

ABSTRACT: From a combination of chiral Mn(salen) struts and the tetratopic ligand tetrakis(4-carboxyphenyl)benzene, a large-pore, noncatenated metal-organic framework (MOF) material, MnSO-MOF, has been synthesized. Following solvent exchange with hydrophobic $\mathrm{CHCl}_{3}$, treatment of MnSO-MOF with aqueous $\mathrm{H}_{2} \mathrm{O}_{2}$ allowed for the selective demetalation of $\mathrm{Mn}$ (salen) struts at and near the surface of the crystals. The resulting crystals displayed greatly enhanced size-selective catalysis compared to the as-synthesized material. Handling of the mechanically fragile MnSO-MOF crystals was greatly facilitated by activation with supercritical $\mathrm{CO}_{2}$.

\footnotetext{
$\Delta \mathrm{s}$ a class of permanently microporous materials, metalAlorganic frameworks (MOFs) have attracted significant attention because of their unique properties. The ability to (a) incorporate a wide range of chemical functionalities via organic struts and (b) control the size and shape of the micropore environment has motivated the synthesis of MOFs for a plethora of applications. ${ }^{1-6}$ In particular, catalysis has emerged as a promising use for MOFs because of the potential for integrating the well-defined, single-site activity of homogeneous catalysts with the shape-, size-, chemo-, and enantioselectivity that can be designed into the micropores. ${ }^{7,8}$ However, tailoring the desired catalysis to occur exclusively within the designed micropores of the MOF crystals, and not on external surface sites, remains a difficult challenge in MOF-based catalysis.

Metallosalen ligands have previously been used as MOF struts by our groups 9 as well as others. ${ }^{10}$ By employing a dipyridyl $\mathrm{Mn}$ (salen) (1) catalyst as a strut in combination with biphenyldicarboxylate (bpdc), a robust, pillared-paddlewheel MOF can be obtained. This MOF $\left[\mathrm{Zn}_{2}(\text { bpdc })_{2}(1)\right](2)$ is a catenated material with medium-sized pores, comprised of two interwoven frameworks (Scheme 1). Catalytic studies with 2 showed greatly improved stability of the heterogenized catalyst 1 over its homogeneous form in the epoxidation of chromene. This promising result suggests that epoxidations catalyzed by $(\mathrm{Mn})$ salen-containing MOFs can serve as versatile platforms for exploring the fundamental characteristics and scope of MOF-based catalysis, especially given the wide versatility and well-studied reactivity of metallosalens. ${ }^{11}$
}

To make a noncatenated analogue of 2 that also possesses larger pores, we replaced the dicarboxylate linker with the tetracarboxylic acid $3{ }^{12}$ which has been shown to form noncatenated mixed-ligand, zinc paddlewheel MOFs. ${ }^{13-16}$ Indeed, by combining the $\mathrm{Mn}$ (salen) 1 with the octaoxygen ligand 3 in the presence of $\mathrm{Zn}\left(\mathrm{NO}_{3}\right)_{2} \cdot 6 \mathrm{H}_{2} \mathrm{O}$, a large-pore crystalline $\mathrm{MOF}$ [MnSO-MOF, $\mathrm{Zn}_{2}$ (3) (1)] can be obtained (Scheme 1). Singlecrystal X-ray diffraction revealed that, unlike 2, MnSO-MOF consisted of a single network with unobstructed channels $(\mathrm{a} \times$ $\mathrm{b}=22.4 \AA \times 11.7 \AA$ ) in comparison to the catenated MOF 2 $(15.7 \AA \times 6.2 \AA)$. Thermogravimetric analysis (TGA) confirmed this high degree of porosity: $\sim 55 \%$ of the as-synthesized material is solvent by weight [Figure S2 in the Supporting Information (SI)].

MnSO-MOF shows excellent activity for the epoxidation of 2,2-dimethylchromene (Figure 1), functioning for nearly 4000 turnovers, stopping only because of depletion of the oxidant [see the SI for safety warnings regarding the synthesis of the 2-(tertbutylsulfonyl)iodosylbenzene oxidant]. However, unlike 2, catalysis by as-synthesized MnSO-MOF crystals displays a significant induction period. We attribute this to diffusion-limited transport of the substrate and/or oxidant through the MOF micropores, which is not observed for 2 because of the significantly smaller sizes of its crystals $(\sim 0.1 \mathrm{~mm} \times 0.1 \mathrm{~mm}$ plates $)$ versus MnSO-MOF $(\sim 1 \mathrm{~mm} \times 0.3 \mathrm{~mm}$ plates). (Recall that diffusion times increase as the square of the distance traversed.)

Intriguingly, treatment of MnSO-MOF with $\mathrm{H}_{2} \mathrm{O}_{2}$ in a water/ methanol solution caused significant depletion of the $\mathrm{Mn}$ content. Overnight treatment reduced the $\mathrm{Mn} / \mathrm{Zn}$ ratio from $\sim 0.5$ in the as-synthesized material to $\sim 0.05$, as observed by inductively coupled plasma optical emission spectroscopy (ICP-OES) of digested samples. This $90 \%$ depletion of the Mn content of the MOF was accompanied by a dramatic color change in the crystals, from dark brown to bright yellow, suggesting retention of the salen ligands despite the loss of $\mathrm{Mn}^{3+}$ ions. Presumably, the $\mathrm{Mn}^{3+}$ ions can be further converted to a soluble manganate species, which can then diffuse out of the MOF. Powder X-ray diffraction reveals that the depleted material still retains some crystallinity, although many features in the pattern disappear (see the SI). TGA and $\mathrm{N}_{2}$ adsorption data (see the SI) of the Mndepleted material after $N, N$-dimethylformamide (DMF) resolvation confirm that it is still porous, although with a reduced solvent

Received: September 23, 2010

Published: March 07, 2011 
Scheme 1. Syntheses of Doubly Interpenetrated (Left) and Noncatenated (Right) Pillared-Paddlewheel MOFs from Dipyridyl Mn(salen) Catalyst $\mathbf{1}^{a}$

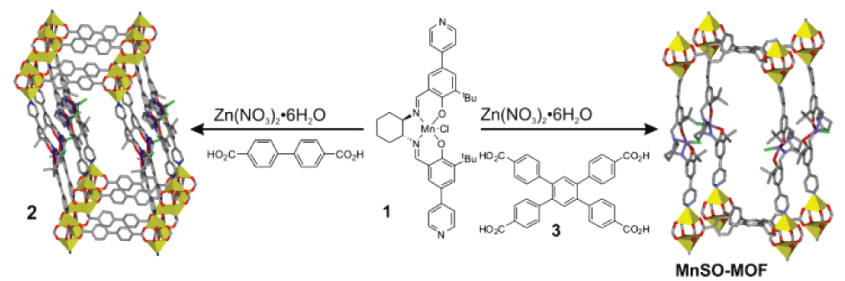

${ }^{a}$ The unit cells of the two MOFs are shown as stick representations (yellow polyhedra $=\mathrm{Zn}$, red $=\mathrm{O}$, green $=\mathrm{Cl}$, blue $=\mathrm{N}$, gray $=\mathrm{C}$, purple $=$ $\mathrm{Mn})$. Solvent molecules, hydrogen atoms, and disordered atoms have been omitted for clarity.

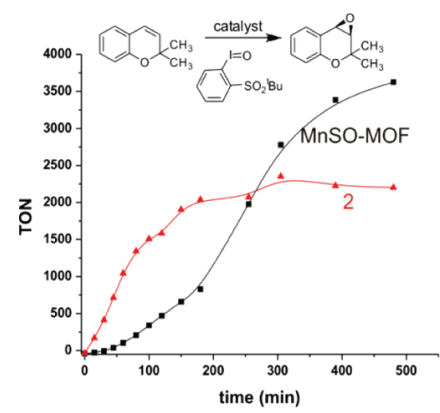

Figure 1. Research profile for the epoxidation of 2,2-dimethylchromene by MnSO-MOF and 2.
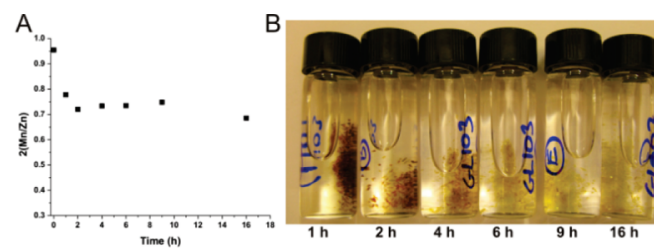

C

D
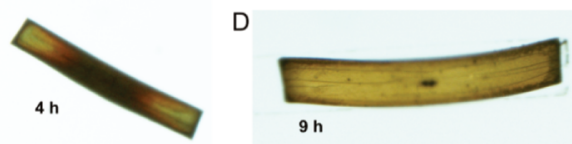

Figure 2. (A) $\mathrm{Mn} / \mathrm{Zn}$ ratio of $\mathrm{H}_{2} \mathrm{O}_{2}$-treated $\mathrm{MnSO}-\mathrm{MOF}$ as a function of the treatment time. (B) Photographs of crystals of MnSO-MOF that have been exposed to aqueous $\mathrm{H}_{2} \mathrm{O}_{2}$ for various amounts of time. ( $\mathrm{C}$ and D) Images of individual crystals of $\mathrm{H}_{2} \mathrm{O}_{2}$-treated MnSO-MOF. Both crystals are $\sim 1 \mathrm{~mm}$ in length.

capacity and surface area. This is unsurprising if the framework remains intact after demetalation but loses some rigidity; freebase salens are known to be significantly less rigid than metalated ligands. $^{17}$

We were thus excited by the possibility of replacing DMF in the MnSO-MOF micropores with a hydrophobic solvent and selectively modifying the surface and near-surface regions of the crystals using aqueous $\mathrm{H}_{2} \mathrm{O}_{2}$. A similar strategy was exploited by our groups to tailor MOF crystals, ${ }^{15,18}$ and the use of $\mathrm{H}_{2} \mathrm{O}_{2}(\mathrm{aq})$ here can allow us to passivate the catalytic sites on the MOF surface and maximize the pore-based activity. Indeed, soaking MnSO-MOF in $\mathrm{CHCl}_{3}$ for several days, followed by treatment with $\mathrm{H}_{2} \mathrm{O}_{2}$, allowed for its systematic $\mathrm{Mn}$ demetalation.

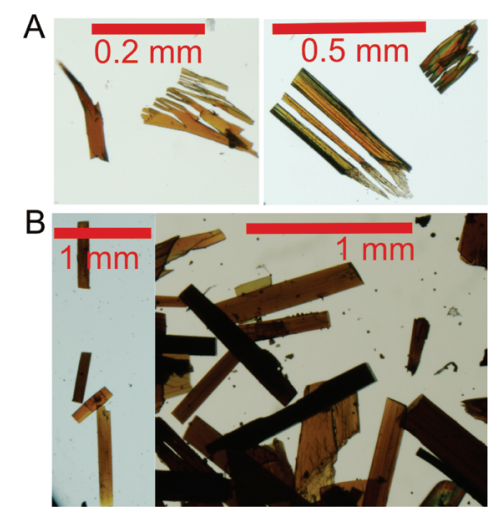

Figure 3. (A) Images of methanol-soaked crystals of MnSO-MOF that have been allowed to dry in air, showing significant fragmentations. (B) Images of crystals of MnSO-MOF that have been activated using supercritical $\mathrm{CO}_{2}$, showing excellent preservation of crystal morphology.

Scheme 2. Size-Selective Catalytic Epoxidation

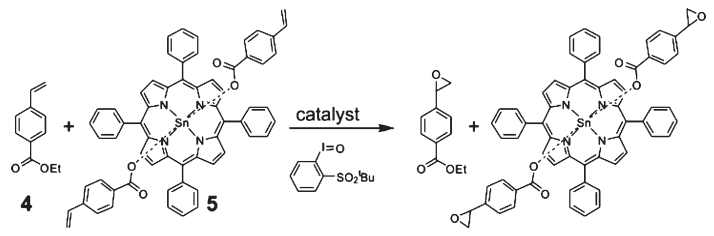

The extent of $\mathrm{Mn}$ demetalation could be controlled by altering the amount of time that MnSO-MOF was exposed to $\mathrm{H}_{2} \mathrm{O}_{2}(\mathrm{aq})$. Metal removal was easily observed via a change in the color of the crystals, from the dark brown of the $\mathrm{Mn}$ (salen) strut to the bright yellow of the demetalated species (Figure 2C,D). In spite of the progressive color change in the crystals, ICP-OES analysis of the treated MOF samples revealed little change in the Mn content after a rapid initial decrease (Figure $2 \mathrm{~A}$ ).

Despite the large size of the MnSO-MOF crystals, their handling is difficult because of high fragility. In particular, accurate weighing of the MOF samples for catalytic experiments was hampered by the tendency of the crystals to fragment when not under a layer of solvent (Figure 3A). This can be a debilitating impediment to any attempt to selectively alter the surface of the crystals: fragmentation would expose the interior of the crystals, and hence new surfaces, to the bulk solution. We hypothesized that if the stresses associated with solvent evaporation could be removed, MnSO-MOF crystal fragmentation would be eliminated. Thus, we subjected crystals of MnSOMOF to supercritical $\mathrm{CO}_{2}$ drying, a process that has been found to prevent both pore collapse in MOF crystals and macroscopic aggregation of crystallites that can accompany evaporation of the liquid solvent. ${ }^{19-23} \mathrm{We}$ were pleased to observe complete preservation of the original as-synthesized crystal morphology (Figure 3B).

To study the effects of selective surface demetalation in MnSO-MOF upon catalytic selectivity, we examined the competitive oxidation of a pair of differently sized styrene derivatives (Scheme 2) using ${ }^{1} \mathrm{H}$ NMR spectroscopy. The small substrate, 4, can easily enter the micropores of the MOF and access interior catalytic sites. The larger substrate, $\mathbf{5}$, should only react with the catalytic sites on the exterior of the crystal. Using as-synthesized 


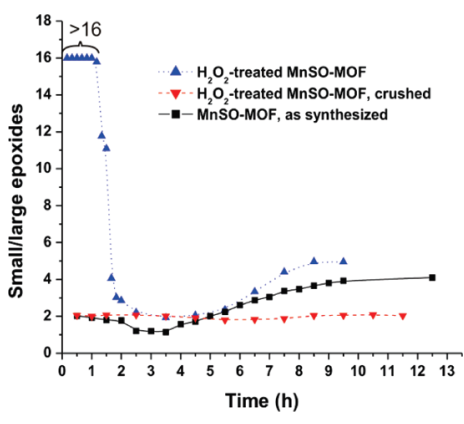

Figure 4. Observed size selectivity for epoxidation of $\mathbf{4}$ and 5 by assynthesized and surface-demetalated MnSO-MOF. At short times, the achievable signal-to-noise ratio allowed only lower limits for small/large reactant selectivity to be determined.

MnSO-MOF, a slight induction period was observed for epoxidation of the small substrate 4 (Figure S4 in the SI), similar to that observed for 2,2-dimethylchromene (Figure 1), implying that catalysis is occurring in the interior of the MOF crystals. Catalysis with a ground-up sample of MnSO-MOF did not exhibit an induction period (Figure S4 in the SI), further confirming that the induction period was due to the large size of the original crystals. As expected for a mixture of surface and internal catalysis, the unmodified MnSO-MOF showed poor size selectivity early in the reaction, and only gradually increased to moderate selectivity ( $4: 1$ in favor of 4 ) as the reaction proceeded. This is qualitatively similar to previous observations for the catenated MOF $2^{9}$ and can be ascribed to the gradual demetalation of surface sites by the oxidant.

A sample of MnSO-MOF that had been treated with aqueous $\mathrm{H}_{2} \mathrm{O}_{2}$ for $4 \mathrm{~h}$ was then examined in the aforementioned sizeselective epoxidation (Scheme 2). This surface-treated MOF did indeed show high selectivity $(\geq 16)$ for the small substrate in the early stages of the reaction (Figure 4 and Figure S5 in the SI). Unfortunately, this selectivity dissipated to $\sim 2: 1$ as the reaction progressed. This behavior was consistent with fragmentation of the large MOF crystals during the course of the reaction, which then exposed new surface catalytic sites to the bulk solution. To test this hypothesis, catalysis was carried out with a sample of the $\mathrm{H}_{2} \mathrm{O}_{2}$-treated Mn-SOMOF that was manually crushed: a constant low selectivity of 2:1, similar to the ratio seen in the later stages of the epoxidation catalyzed by the large crystals, was indeed observed (Figure 4).

In conclusion, we have demonstrated that a noncatenated Mn(salen)-based MOF can be treated with aqueous $\mathrm{H}_{2} \mathrm{O}_{2}$ to selectively deactivate surface catalytic sites, thus reducing the surface and nearsurface reactivity. Furthermore, we have shown that treatment with supercritical $\mathrm{CO}_{2}$ is an effective strategy for eliminating crystal fragmentation (and interior site exposure) during activation. By application of both strategies, high substrate size selectivity can be engendered for epoxidation catalysis by MnSO-MOF, albeit only for a limited time. The synthesis of metallosalen MOFs with large pores will allow for the future exploration of a wide variety of catalytic reactions, especially those that require significantly larger pore environments than have been previously available.

\section{ASSOCIATED CONTENT}

S Supporting Information. Synthesis and characterization data for MnSO-MOF including single-crystal XRD data in CIF format, an improved synthesis of $\mathbf{2}$, details of the supercritical $\mathrm{CO}_{2}$ processing procedure, a description of the catalytic conditions and full catalytic results, including safety warnings and enantioselectivity data. This material is available free of charge via the Internet at http://pubs.acs.org.

\section{AUTHOR INFORMATION}

\section{Corresponding Author}

*E-mail: j-hupp@northwestern.edu (J.T.H.); stn@northwestern. edu (S.T.N.).

\section{ACKNOWLEDGMENT}

We gratefully acknowledge the U.S. Department of Energy (Grant DE-FG02-03ER15457), DTRA, Northwestern NSEC, AFOSR, (agreement no. FA9550-07-1-0534) for financial support of this work.

\section{REFERENCES}

(1) Feréy, G. Chem. Soc. Rev. 2008, 37, 191-214.

(2) Li, J.-R.; Kuppler, R. J.; Zhou, H.-C. Chem. Soc. Rev. 2009, $38,1477-1504$

(3) Murray, L. J.; Dinca, M.; Long, J. R. Chem. Soc. Rev. 2009, $38,1294-1314$

(4) Tranchemontagne, D. J.; Mendoz-Cortes, J. L.; O'Keeffe, M.; Yaghi, O. M. Chem. Soc. Rev. 2009, 38, 1257-1283.

(5) Allendorf, M. D.; Bauer, C. A.; Bhakta, R. K.; Houk, R. J. T. Chem. Soc. Rev. 2009, 38, 1330-1352.

(6) Chen, B.; Xiang, S.; Qian, G. Acc. Chem. Res. 2010, $43,1115-1124$.

(7) Lee, J.-Y.; Farha, O. K.; Roberts, J.; Scheidt, K. A.; Nguyen, S. T.; Hupp, J. T. Chem. Soc. Rev. 2009, 38, 1450-1459.

(8) Ma, L.; Abney, C.; Lin, W. Chem. Soc. Rev. 2009, 38, 1248-1256.

(9) Cho, S.-H.; Ma, B.; Nguyen, S. T.; Hupp, J. T.; Albrecht-Schmitt, T. E. Chem. Commun. 2006, 2563-2565.

(10) Kitaura, R.; Onoyama, G.; Sakamoto, H.; Matsuda, R.; Noro, S.-i.; Kitagawa, S. Angew. Chem., Int. Ed. 2004, 43, 2684-2687.

(11) Katsuki, T. Synlett 2003, 281-297.

(12) Farha, O. K.; Mulfort, K. L.; Hupp, J. T. Inorg. Chem. 2008, 47, 10223-10225.

(13) Mulfort, K. L.; Farha, O. K.; Stern, C. L.; Sarjeant, A. A.; Hupp, J. T. J. Am. Chem. Soc. 2009, 131, 3866-3868.

(14) Shultz, A. M.; Farha, O. K.; Hupp, J. T.; Nguyen, S. T. J. Am. Chem. Soc. 2009, 131, 4204-4205.

(15) Gadzikwa, T.; Farha, O. K.; Malliakas, C. D.; Kanatzidis, M. G.; Hupp, J. T.; Nguyen, S. T. J. Am. Chem. Soc. 2009, 131, 13613-13615.

(16) During the writing of this manuscript, another report of noncatenated metallosalen MOFs appeared: Song, F.; Wang, C.; Falkowski, J. M.; Ma, L.; Lin, W. J. Am. Chem. Soc. 2010, 132, 15390-15398.

(17) Sun, S.-S.; Stern, C. L.; Nguyen, S. T.; Hupp, J. T. J. Am. Chem. Soc. 2004, 126, 6314-6326.

(18) Gadzikwa, T.; Lu, G.; Stern, C. L.; Wilson, S. R.; Hupp, J. T.; Nguyen, S. T. Chem. Commun. 2008, 5493-5495.

(19) Nelson, A. P.; Farha, O. K.; Mulfort, K. L.; Hupp, J. T. J. Am. Chem. Soc. 2009, 131, 458-460.

(20) Bae, Y.-S.; Dubbeldam, D.; Nelson, A. P.; Walton, K. S.; Hupp, J. T.; Snurr, R. Q. Chem. Mater. 2009, 21, 4768-4777.

(21) Cooper, A. I.; Rosseinsky, M. J. Nat. Chem. 2009, 1, 26-27.

(22) Farha, O. K.; Hupp, J. T. Acc. Chem. Res. 2010, 43, 1166-1175.

(23) Tsao, C. S.; Chen, C. Y.; Chung, T. Y.; Su, C.J.; Su, C. H.; Chen, H. L.; Jeng, U. S.; Yu, M. S.; Liao, P. Y.; Lin, K. F.; Tzeng, Y. R. J. Phys. Chem. C 2010, 114, 7014-7020. 